\title{
Erratum to: Identification of Cross-Section Defects of the Rod by Using Eigenfrequencies and Features of the Shape of Longitudinal Oscillations
}

\author{
L. D. Akulenko ${ }^{a, b}$, A. A. Gavrikov ${ }^{a, *}$, and S. V. Nesterov ${ }^{a}$ \\ ${ }^{a}$ Ishlinsky Institute for Problems in Mechanics, Russian Academy of Sciences, \\ pr. Vernadskogo 101, str. 1, Moscow, 119526 Russia \\ ${ }^{b}$ Bauman Moscow State Technical University, ul. 2-ya Baumanskaya 5, Moscow, 105005 Russia \\ *e-mail:gavrikov@ipmnet.ru \\ Received May 20, 2020
}

DOI: $10.3103 / \mathrm{S} 0025654420010227$

The Section Acknowledgements should read "The work was carried out on the topic of state assignment (no. of state registration AAAA-A17-117021310381-8, AAAA-A17-117021310387-0) and in part by Russian Foundation for Basic Research (no.18-01-00812 A).”

The original article can be found online at https://doi.org/10.3103/S0025654419080119 